\title{
Weighted Fuzzy Rule Based Sentiment Prediction Analysis on Tweets
}

\author{
Syed Muzamil Basha, Yang Zhenning, Dharmendra Singh Rajput*, \\ Iyengar N.Ch.S.N and Ronnie D. Caytiles ${ }^{\#}$ \\ School of Computer Science and Engineering.VIT University, Vellore, T.N., India \\ "School of Information Technology and Engineering, VIT University, Vellore, T.N., India \\ ${ }^{\#}$ Multimedia Engineering department, Hannam University, Daejeon, Korea \\ muza.basha@gmail.com,yang.zhenning2015@vit.ac.in, \\ dharmendrasingh@vit.ac.in,nchsniyengar48@gmail.com,rdcaytiles@gmail.com
}

\begin{abstract}
As E-Commerce is becoming more popular, the number of product reviews that a product received grows exponentially. In this context, others' opinions will play a vital role to make a decision to select among multiple options involves valuable resources like money and time, where people usually depends on their peers' past experiences in the form of reviews. Many companies use opinion mining and sentiment analysis as part of their research. Detecting sentiment in social media like Facebook, Twitter, online blogs and reviews have become an essential task as they have been influencing every business organization. In this paper, we would like to analyze the Fuzzy rule-based systems (FRBSs) with FRBS models, namely, Mamdani and Takagi Sugeno Kang (TSK) using FRBS package in $R$, and a comparison with other common classification methods. Additionally, the performance of FRBSs method is measured in terms of precision, Recall and F-measure to find accuracy in classification.
\end{abstract}

Keywords: opinion mining, sentiment analysis, Fuzzy Rule based sentiment prediction

\section{Introduction}

This instruction file for Word users In the era of social connectedness, people are becoming increasingly enthusiastic about interacting, sharing, and collaborating through social networks, online communities, blogs, Wikis, as well as a range of other online collaborative media. Among which Twitter is one, where Twitter is an online social networking service that allows users to post and read short messages, called "tweets". Each tweet is 140 characters in Length. 240+ million active users, 500 million Tweets are generated every day, users often discuss current affairs and share personal views on various subjects, Tweets are used to express emotions' on particular subject.

The attributes of Tweets are: length of a Tweet, Data availability, Language model and Domain. The problem in sentiment Analysis on Tweets is classifying the polarity of a given text at the document, sentence and feature level and to determine whether the expressed opinion in a document, sentence and feature is positive, negative,(or) neutral. Most common thought is the expression of personal feelings through Social Networking Services (SNS's) in evaluating the services and products. A wide variety of applications can benefit from such approaches like: marketing groups can receive and evaluate feedback from a large set of users, politicians can estimate their popularity, and manufacturers can identify the drawbacks of their products, while the common users are facilitated to navigate the huge online reviews. The existing techniques to derive the sentiment from reviews are language dependent: they are designed for particular language, usually depends on a dictionary (Word-Net7) for assessing the meaning or the lexical category of specific words or phrases. The textual content of Social Media, 
however, breaks the fundamental assumption of these methods, due to its inherent characteristics. The textual content of Social Media is rather informal, since the communication principally comprises between related people. Therefore, common users employ words and expressions that do not have any standard in any language. For example, "n8" is commonly used instead of "night" and "G8" instead of "Great". As a result, the user-generated text contains misspelled words and incorrect use of grammar. Goals of analyzing sentiments from natural language text will lead to more bio-inspired approaches, to design the intelligent opinion-mining systems capable of handling semantic knowledge, making analogies, learning new effective knowledge, and detecting, perceiving, and "feeling" emotions.

In this paper, we evaluate the performance of Fuzzy Rule based approach while classifying sentiments from tweets. Using the Tweeter Corpus consisting of 50,000 tweets, we will evaluate our results by comparing against Term Based Approach. The feature extractors used in our research are n-grams, Lexicon for emotion extraction and worked on combination of different emotions Identified and plotted the same in the result section. In addition to that we have train the model using Machine learning models as discussed in detail in the below sections and measure the performance of the same using precision, recall and F-score. Finally, we use cross validation method to find the mean accuracy in classification.

Our paper is organized as, Section 2 deals problem formulation, Section 3 gives idea on research carried out in the field of classification sentiment, In section 4 describe the way the research is carried out in our paper as Methodology, In Section 5 we discussed the way the rules are constructed to perform sentiment analysis using Fuzzy Logic with manually assigned weights based on the impact of each emotion present at Tweet level. In Section 6 discussion on Machine Learning algorithms used for classification. Finally, Section 7 we plotted our results that are obtained in form of graphs with combination of different emotions considered in our experiment. In Section 8 we evaluated the performance of the Machine Learning algorithms used in classification of sentiments using parameters precision, recall and F-score. In Section 9 we perform the validation of our model using cross validation method. In section 10 we discussed the way the results are obtained and finally we concluded along with future scope left out in the area of Fuzzy Rule based sentiment classification.

\section{Problem Formulation}

Based on literature made, the Sentiment analysis is typically modeled as binary classification problem as shown in the equation 1 .

\section{1: Exact scored : all the words in tweets are perfectlyscored 0:Not Scored: Nothing or only a word intweets are prefectlyscored}

This assumption simplifies the problem of sentiment analysis, resulting in higher classification accuracy. In real world sentiment analysis applications rarely consists of just polarized tweets. So, different methods have to be considered, to address the additional class of neutral tweets as shown in equation 2 .

$$
\begin{aligned}
& 1: \text { Exact scored }: \text { all the words in tweets are perfectlyscored } \\
& \alpha: \text { AdditionalNon-scored words are present in the tweet } \\
& \gamma \times \frac{n}{N}: \text { Incompletescoring }: \text { only } n>1 \text { of } N \text { scored words } \\
& 0: \text { Not Scored }: \text { Nothing oronly a word intweets are prefectlyscored }
\end{aligned}
$$


$0 \leq \alpha \leq 1$ and $0 \leq \gamma \leq 1$ Parameters are used to measure imperfect scored words in each tweet present in the document. Since the patterns we used to represent in social media are relatively short, exact matches are uncommon, and taking advantage of partial matches allows us to significantly improve the accuracy of classification. Cambria et al., (2013) stated that Opinion mining mainly focuses on semantic inferences and effective information associated with natural language, and it does not require a deep understanding of the text, sentiment analysis, research moving toward context-based analysis of natural language text, supported by time efficient parsing techniques suitable for big social data analysis. Gouws et al., (2011) made a study on possible different types of transformations that are common between user contexts.

\section{Related Work}

In our Literature work, our attempt is to identify the amount of research carried out in the proposed area and the gap left in the same. As part of that, Aisopos et al., (2011) used n-gram graph model to capture patterns effectively in the polarized, textual content of Social Media, conducted a comparative analysis between n-gram graphs and vector model. Bollen et al., (2011b) investigates whether public sentiment can be used to predict the stock market, used tools like: Opinion Finder and GPOMS to measure variations in the public mood from tweets submitted on a daily basis. Bravo-Marquez et al., (2016) made a study how to compute our word-level sentiment association attributes from tweets annotated with both hard and soft labels obtained from different collections of automatically labeled tweets. Chen et al., (2015) made an analysis on individual behavior rather than the micro-blogs created by an individual user, considered original words and retweet others' tweets for predicting future vitality. Alahmadi and Zeng (2015) had proposed Implicit Social Trust and Sentiment (ISTS) based Re-commander System RS on Online Social Networks (OSNs) by utilizing the implicit trust between friends and the sentiment they hold in their posts, also employed a probabilistic method to support the illustration of the intensity of sentiment in micro-reviews into numerical rating scales. Jin et al., (2016) stated that product features and sentiment polarity are first to be identified from online reviews with the help of positive and negative reviews, developed an approach based on conditional random fields (CRFs) to label each word in online reviews.

Kumar and Ravi (2016) have made a comprehensive review of various financial applications in Text mining like: FOREX Rate Prediction, Stock Market Prediction, Customer Relationship Mangement (CRM) subsuming Churn Prediction, and cyber security subsuming Phishing Detection, Spam Detection, Malware Detection, Fraud Detection and Intrusion Detection. Finally, summarizing all the applications associated with automatic sentiment analysis along with challenges for automatic systems and algorithms, features, and data sets used in sentiment analysis by Mohammad (2016).

\section{Methodology}

In our approach, At one end text is given as input to the system and at the other end some market predictive values are expected as output, the same is projected in the Figure 1. 


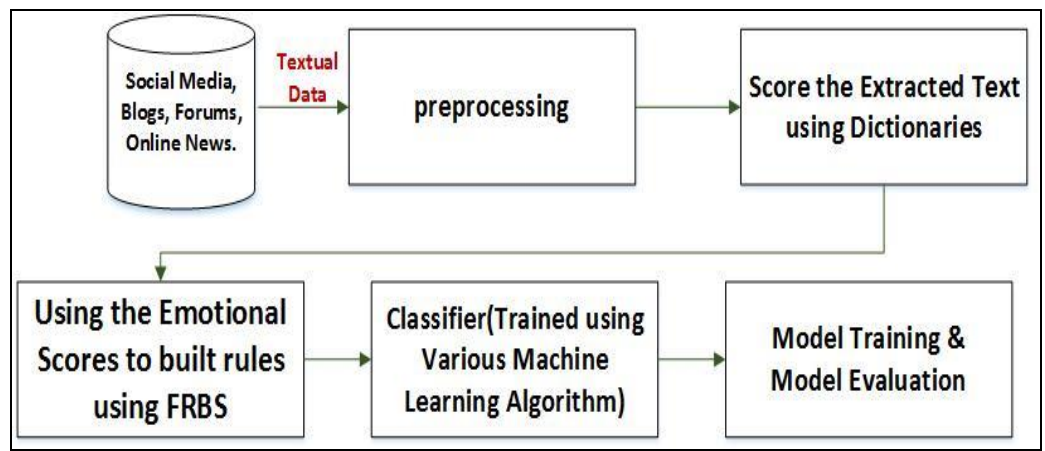

\section{Figure 1. Basic Frame Work of Sentiment Analysis on Product Reviews from Tweets}

\subsection{Input Dataset}

To consider the Input Source data, first step is to understand the behavior of the attributes present in it, as an attempt to understand the same, we focused on Riza et al., (2015) proposed a fast and systematic approach, called Fast Lead User Identification (FLUID), utilizing data mining techniques to effectively identify lead users of the microblogging site Twitter. Mate et al., (2016) proposed a hybrid architecture that is able to integrate on the fly highly heterogeneous data sources, combined with contextual data which are integrated with a variety of information sources or Big Data, make some predictions about energy consumption based on energy data mining. The source of data collected from repositories may be in two from calling Text data and Market data. Bollen et al., (2011a) has focused on twitter 9,853,498 Tweets and utilize it for market prediction and public mood analysis.

\subsection{Preprocessing}

Following are the steps performed to clean the data in our experiments.

\section{Preprocessing}

Step 1: Removing Re-Tweets, HTTP Links Names of the people, unnecessary spaces and punctuations' which are not considered suitable for evaluating the sentiment score.

Step 2: The text in the body section is split into sentences using sentence Tokenizer.

Step 3: Stop words are removed since they are not useful in discriminating between relevant and irrelevant sentences.

Step 4: Stemming process is performed on all remaining terms in tweet.

There are at three sub-processes of pre-processing namely: feature-selection, dimensionality-reduction, feature-representation.

4.2.1. Feature-selection: In Decision Making process, the selection of features like: Terms presence and frequency, Parts of speech(POS), Opinion words and phrases and Negations through which required text is to be represented is very important, because from an incorrect representation of data(Text and Market) input nothing more than a meaningless output is generated. Vu et al. (2012) considered a Daily aggregate number of positives or negatives on Twitter Sentiment Tool (TST) and an emoticon lexicon. Daily mean of Pointwise Mutual Information (PMI) for pre-defined bullish-bearish anchor words. Bollen et al., (2011a) used Opinion Finder tool for future selection. Lugmayr and Gossen (2013) considered Bag-of-words.

4.2.2. Dimensionality-Reduction: Limiting the number of features is next important as the more in the number of features, make the classification problem extremely difficult to 
solve that leads in decreasing the efficiency of Machine Learning Algorithms. Vu et al. (2012) Predefined company related keywords, Named Entity Recognition based on linear conditional random fields (CRF). Bollen et al., (2011a) used Opinion Finder tool for dimensionality-reduction. Lugmayr and Gossen (2013) performed Stemming.

4.2.3. Feature-Representation: With the minimum number of features determined in the previous step, each feature needs to be represented in the form of a numeric value. Which acts as inputs to machine learning algorithms for classification. Vu et al., (2012) Real number for Daily NegP os and BullishBearish. Bollen et al., (2011a) represented feature using Opinion Finder, Lugmayr and Gossen (2013) represented has Sentiment Value.

\subsection{Different Emotion Scoring Techniques}

Generally, emotions are indicated using cardiovascular measures Mauss and Robinson (2009) has given mostcommonly used cardiovascular indicators like: heart rate (HR), blood pressure (BP), total peripheral resistance (TPR), cardiac output (CO), pre-ejection period (PEP), and heart rate variability (HRV)as measure of Emotions. Bollen et al., (2011a) has maps Profile of Mood States function(POMS) P(t) of each tweet to a sixdimensional mood vector $\mathrm{m}$ TM6. The entries of $\mathrm{m}$ represent the following six dimensions of $\operatorname{mood}(\mathrm{TM})$ : Tension, Depression, Anger, Vigour, Fatigue, and Confusion. The POMS-scoring function $\mathrm{P}(\mathrm{t})$ simply matches the terms extracted from each tweet to the set of POMS mood adjectives for each of POMS' 6 mood dimensions. Herring (2011) focused on structural linguistic process, and approaches, such as machine learning models that can identify the syntax and semantic of Tweets through computer-mediated discourse analysis.

Khan et al., (2015) used opinion lexicon to score a given sentence consist of the usergiven entity, in which all opinion words from the sentence are identified by matching with the words in the opinion lexicon. Finally, computed an orientation score for the sentiment. A positive word is assigned the semantic orientation score of +1 and a negative word is assigned the semantic orientation score of -1 . All the scores are then summed up as shown in the equation 3.

$$
\operatorname{score}(s)=\sum_{w_{i}: w_{i} \in L \cap w_{i} \in s} \frac{w_{i} \bullet \text { score }}{d\left(w_{i}, s\right)}
$$

where wi is opinion word Le is opinion lexicon, $\mathrm{S}$ is the sentence that contains the sentiment $s$ and $d\left(w_{i} ; s\right)$ is the distance between the sentiment words in particular tweet. Abbott et al., (2011)analyzed the usage of meta-post features, contextual features, dependency features and word-based features for identifying the disagreement relation among participants, used contextual and dialogic features and achieved accuracy up to $68 \%$ as compared to a unigram baseline of $63 \%$ by derive cosine similarity measure using WF \& DF vectors. In which, each distinct term as one feature and calculate a Word Frequency (WF) value for each feature. The WF weight for term $i$ in post $\mathrm{j}$ is evaluated as shown in equation 4 :

$$
w f=\frac{n_{i j}}{\sum_{k} n_{k j}}
$$

where $n_{i j}$ is the number of occurrences of term $i$ in post $j$, and the denominator is the count of the occurrences of all terms in post $j$. Wf will be larger for terms that appear most frequently in the tweet. The Document Frequency (DF) provides a measure of how important a particular term is within the entire corpus. DF for term $i$ is defined as: 


$$
D F=\log \frac{|T|}{t_{j}: w_{i} \in t_{j}}
$$

where $|\mathrm{T}|$ is the total number of tweets in a dataset, $\mathrm{t}_{\mathrm{j}}$ is the number of posts in which term $\mathrm{w}_{\mathrm{i}}$ appears. DF scores are higher for terms which are good discriminators between posts. Thus, each tweet is represented as a vector of words and each word is represented in the vector by its WFDF value. Terms that appear in the corpus but not in a given post will receive a WFDF weight of 0 (because WF will be 0 ) as shown in the equation 6 .

$$
W F D F=W F_{i j} \times D F_{i}
$$

Balasubramanyan et al., (2011) discussed different approaches to identify the emotions in posts like: making use of SentiWord Net, principle of point wise mutual information. Agarwal et al., (2011) considered dictionary of about 8000 English language words assigns every word a pleasant score between 1 (Negative) 3 (Positive), then normalized the scores by diving each score my the scale 3 and consider words with polarity less than 0:5 as negative, higher than 0:8 as positive and the rest as neutral. Kouloumpis et al., (2011) investigates the use of linguistic features like: uni-grams and bi-grams lexicon, POS and some features of domain-specific language of micro blogging for detecting the sentiment from Tweets posts'. Khan et al., (2014) proposed Polarity Classification Algorithms like: Enhanced Emoticon Classifier (EEC), Improved Polarity Classifier (IPC) and SentiWordNet Classifier (SWNC) in Twitter Opinion Mining.

\section{Fuzzy Rule Based System}

Fuzzy Logic Design application in MATLAB is for building fuzzy inference systems and viewing and analyzing results. In which Membership functions for creating fuzzy inference systems that Support for AND, OR, and NOT logic in user-defined rules. The Standard Models Mamdani and Sugeno-type fuzzy inference systems. This application also provide facility to Automate membership function shaping through neuroadaptive and fuzzy clustering learning techniques. It has ability to embed a fuzzy inference system in a Simulink model and to generate embeddable $\mathrm{C}$ code or stand-alone executable fuzzy inference engines.

The idea (Mohammad SM et .al., 2013) is to use the dictionary/lexicon containing lots of words with associated scores for eight different emotions and two sentiments (positive/negative). Each individual word in the lexicon will have a "yes" (one) or "no" (zero) for the emotions and sentiments, and we have calculate the total sentiment of a sentence by adding up the individual sentiments for each word in the sentence. FRBS models but also to implement the most widely used learning procedures for FRBSs as shown in Table 1 and phases in Figure 2.

Table 1. Summary on Different Emotions

\begin{tabular}{|c|c|c|c|c|c|}
\hline \multirow{2}{*}{ Sentiment Type } & \multicolumn{5}{|c|}{ Name of the Dataset(Total Tweets) } \\
\cline { 2 - 6 } & $\begin{array}{c}\text { auto } \\
(2014)\end{array}$ & $\begin{array}{c}\text { baby } \\
(1128)\end{array}$ & $\begin{array}{c}\text { baby5 } \\
(965)\end{array}$ & $\begin{array}{c}\text { pet } \\
(417)\end{array}$ & $\begin{array}{c}\text { product } \\
(2220)\end{array}$ \\
\hline Anger & 1382 & 797 & 518 & 160 & 631 \\
\hline Anticipation & 2664 & 1967 & 1474 & 547 & 2819 \\
\hline Disgust & 638 & 943 & 370 & 146 & 426 \\
\hline Fear & 949 & 895 & 571 & 202 & 628 \\
\hline Joy & 1978 & 2360 & 2028 & 557 & 3049 \\
\hline
\end{tabular}




\begin{tabular}{|c|c|c|c|c|c|} 
Sadness & 1050 & 1181 & 792 & 200 & 907 \\
\hline Surprise & 1225 & 853 & 675 & 264 & 1176 \\
\hline Trust & 3057 & 2257 & 1893 & 828 & 3323 \\
\hline \multicolumn{7}{|c|}{ Sentiments } \\
\hline Negative & 2895 & 2140 & 1356 & 457 & 1606 \\
\hline Positive & 4661 & 4334 & 3609 & 1307 & 6030 \\
\hline
\end{tabular}

Level of Emotion $=\{$ Low, Medium, High $\}$ the Membership function details are listed in the Table 4 and plotted in figure 3.

Next our attempt is to model the our sentiments: we need to determine the exact sentiments considering some factors such as the number of positive and negative sentiments. So, let us consider nine objects $=$ \{anger, anticipation, Disgust, fear, Joy, Happy, Sad, Surprise, Trust $\}$ with linguistic values as follows: Levels of sentiment = \{Very Negative, Negative, Neutral, Positive, Very Positive\}. Based on a particular condition, we can define a fuzzy IF-THEN rule as follows: IF number of vehicles is small and width of street is medium THEN speed of car is fast. IF anticipation is high and Trust is high the output is Very positive with weight one. where $I_{i j k}$ is input matrix with $i$ rows representing number of rules (Here we have plotted 8 rules only) consructed and $j$ coloums with representing number of emotions $E_{i}=\left\{E_{1}, E_{2}, E_{3}, E_{4}, E_{5}, E_{6}, E_{7}, E_{8}, E_{9}\right\}$ and $k$ is used to represented level $\{1,2,3\}$. $o p_{i j}$ is operation matrix representing type of operation $j=\{\wedge, \vee, \neg\}$ on $i^{\text {th }}$ rule. $w_{i j}$ represents weight matrix assigned to each rule in $I_{i j k}$ which is manually assigned based on the emotions, ranges between $j=[0 \leftrightarrow 1], o_{i j}$ represents the output matrix with five different levels $j=\{1,2,3,4,5\}$.

Table 2. Details of Input/Output Membership Functions

\begin{tabular}{|l|l|}
\hline Details of Input Member Function & Details of output Member Function \\
\hline Name='anticipation' & Name='output1' \\
Range=[0 1] & Range=[0 1] \\
NumMFs=3 & NumMFs=5 \\
MF1='L':'gaussmf',[0.1699 6.939e-18] & MF1='VN':'gaussmf',[0.1062 0] \\
MF2='M':'gaussmf',[0.1699 0.5] & MF2='N':'gaussmf',[0.1062 0.25] \\
MF3='H':'gaussmf',[0.1699 1] & MF3='NE':'gaussmf',[0.1062 0.5] \\
& MF4='P':'gaussmf',[0.1062 0.75] \\
& MF5='VP':'gaussmf',[0.1062 1] \\
\hline
\end{tabular}

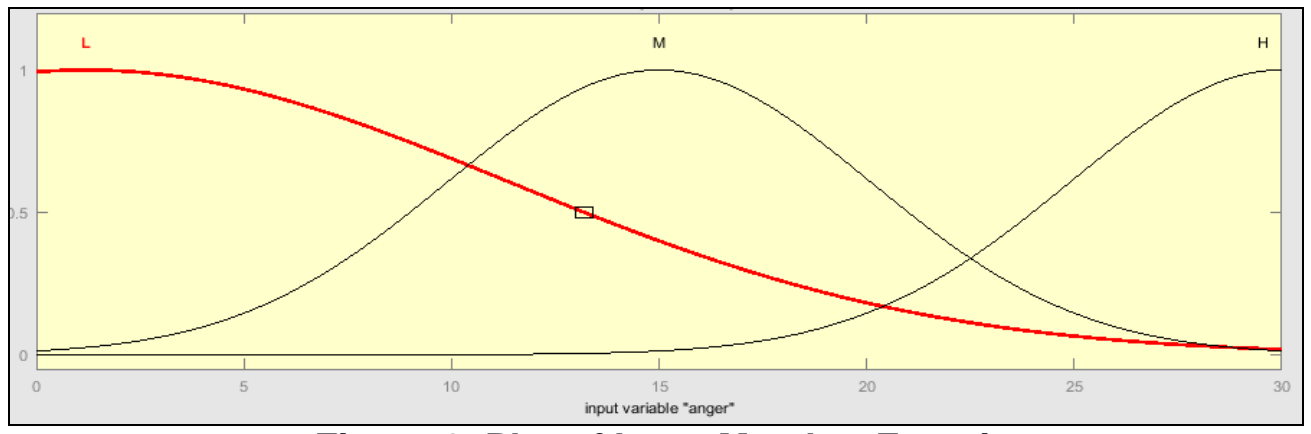

Figure 2. Plot of Input Member Function 
Table 3. Details of Models Implemented

\begin{tabular}{|l|l|}
\hline Mamdani(2.0) & Sugeno(2.0) \\
\hline NumInputs=9 & NumInputs=9 \\
NumOutputs=1 & NumOutputs=1 \\
NumRules=52 & NumRules=50 \\
AndMethod='min' & AndMethod='prod' \\
OrMethod='max' & OrMethod='probor' \\
ImpMethod='min' & ImpMethod='prod' \\
AggMethod='max' & AggMethod='sum' \\
DefuzzMethod='centroid' & DefuzzMethod='wtaver' \\
\hline
\end{tabular}

From the above matrix we can derive the way the rules are constructed and the weights are assigned as shown in equation 7 and 8 .

$$
\begin{gathered}
\left\{\left\{00_{i j}\right\}_{i=1}^{n}\right\}_{j=0}^{3}=\prod_{s=1}^{n}\left\{\left\{\left\{\left\{I_{i j k}\right\}_{i=1}^{n}\right\}_{j=1}^{9}\right\}_{k=1}^{3} \bullet\left\{\left\{r p_{i j}\right\}_{i=1}^{n}\right\}_{j=1}^{3} \bullet\left\{\left\{\left\{I_{i j k}\right\}_{i=1}^{n}\right\}_{j=1}^{9}\right\}_{k=1}^{3}\right\} \Leftarrow\left\{\left\{w_{i j}\right\}_{i=1}^{n}\right\}_{j=0}^{1}(7) \\
\left\{\left\{0{ }_{i j}\right\}_{i=1}^{n}\right\}_{j=0}^{5}=\prod_{s=1}^{n}\left\{\left\{\left\{\left\{I_{i j k}\right\}_{i=1}^{n}\right\}_{j=1}^{9}\right\}_{k=1}^{5} \bullet\left\{\left\{o p_{i j}\right\}_{i=1}^{n}\right\}_{j=1}^{3} \bullet\left\{\left\{\left\{\left\{I_{i j k}\right\}_{i=1}^{n}\right\}_{j=1}^{9}\right\}_{k=1}^{5}\right\} \Leftarrow\left\{\left\{w_{i j}\right\}_{i=1}^{n}\right\}_{j=0}^{1}\right.
\end{gathered}
$$

(Kaya E et. al., 2013) have used Gravitational search algorithm(GSA) to find rule weight values to maximize classification accuracy, followed steps:

1. Generated candidate fuzzy rules.

2. Selected $\mathrm{N}$ fuzzy rules for each consequent class according to selection criterion.

3. Weighted the selected fuzzy rules by using GSA.

4. Created fuzzy rule-based classifier with weighted fuzzy rules.

Table 4. Notations for Different Components

\begin{tabular}{|l|l|l|}
\hline Name of the component & With Three Levels & With Five Levels \\
\hline Input & $\left\{\left\{\left\{\underline{I}_{i j k}\right\}_{\mathrm{i}=1}^{n}\right\}_{\mathbf{j}=1}^{9}\right\}_{\mathbf{k}=1}^{3}$ & $\left\{\left\{\left\{I_{i j k}\right\}_{i=1}^{n}\right\}_{j=1}\right\}_{k=1}^{5}$ \\
\hline Weights & $\left\{\left\{f o p_{i j}\right\}_{i=1}^{n}\right\}_{j=1}^{3}$ & \\
\hline Output & $\left\{\left\{w_{i j}\right\}_{i=1}^{n}\right\}_{j=0}^{1}$ & \\
\hline$\left.\left\{\int_{i j}\right\}_{i=1}^{n}\right\}_{j=0}^{3}$ & $\left\{\left\{0_{i j}\right\}_{i=1}^{n}\right\}_{j=0}^{5}$ \\
\hline
\end{tabular}




$$
\begin{aligned}
& I_{i j k}=\left[\begin{array}{ccccccccc}
E_{1} & E_{2} & E_{3} & E_{4} & E_{5} & E_{6} & E_{7} & E_{8} & E_{9} \\
1 & 1 & 1 & 1 & 1 & 1 & 1 & 1 & 1 \\
3 & 3 & 3 & 3 & 3 & 3 & 3 & 3 & 3 \\
-3 & 3 & 3 & 3 & -3 & -3 & -3 & 3 & 3 \\
-3 & 3 & 3 & 1 & -3 & -3 & -2 & 1 & 1 \\
-2 & 1 & 1 & 1 & -3 & -3 & -2 & 1 & 1 \\
2 & 1 & 1 & 1 & 3 & -3 & -2 & 1 & 1 \\
1 & 1 & 1 & -3 & -3 & -3 & -2 & -3 & 1 \\
1 & 2 & 2 & -1 & -1 & -1 & -1 & -1 & 1
\end{array}\right] \\
& o p_{i j}=\left[\begin{array}{l}
2 \\
2 \\
2 \\
2 \\
2 \\
1 \\
1 \\
1
\end{array}\right] w_{i j}=\left[\begin{array}{c}
1 \\
1 \\
0.8 \\
0.1 \\
0.5 \\
1 \\
0.25 \\
0.5
\end{array}\right] o_{i j}=\left[\begin{array}{c}
1 \\
5 \\
5 \\
4 \\
3 \\
1 \\
2
\end{array}\right]
\end{aligned}
$$

$G(t)=G_{0} e^{-a \frac{t}{T}}$

where $G(t)$ is gravitational constant at time $t, G_{0}$ is initial gravitational constant, $T$ is the total number of iteration.

$$
\begin{aligned}
& \left\{\left\{w_{i j}\right\}_{i=1}^{n}\right\}_{j=0}^{1}=\left(\frac{\operatorname{impact}\left(E_{i t}\right)_{i=1}^{9}}{\sum_{i=1}^{9} \operatorname{impact}\left(E_{T}\right)-E_{i t}}\right) \\
& \operatorname{impact}\left(E_{i t}\right)=\frac{\sum_{i=1}^{l(t)} E_{i}}{\sum_{i=1}^{l(t)}\left(E_{T}-E_{i}\right)}
\end{aligned}
$$

\section{Machine Learning Algorithms}

Support Vector Machine (SVM) Lugmayr and Gossen (2013) implemented the SVM algorithm and used Senti-WordNet software to produce sentiment indicators. Regression Algorithms Hagenau et al., (2013) implemented Regression algorithms considered syntax and semantics for classification. Naive Bayes Cambria et al., (2013) imple-mented Naive Bayes, used Open-source natural language toolkit (NLTK) software for classification. Decision Tree Vu et al., (2012) implemented C4.5 Decision tree, trained by previous day features, used software's like: CRF++ toolkit, Firehose, TST, CMU POS Tagger, AltaVista. 


\section{Result}

Model was trained using Fuzzy Designer tool in Mat Lab, Type of FRBS model " mamdani ", and " sugeno " Type of membership functions: "GAUSSIAN", Type of tnorm method: "Standard t-norm (min)", Type of s-norm method: "Standard s-norm", Type of implication function: "ZADEH", we obtain number of rules given in Table 2. In Figure 4 we plotted the degree of rules obtained using FRBCS.

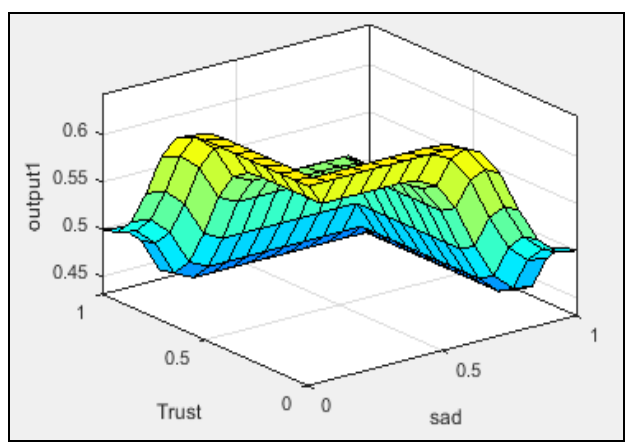

(a)

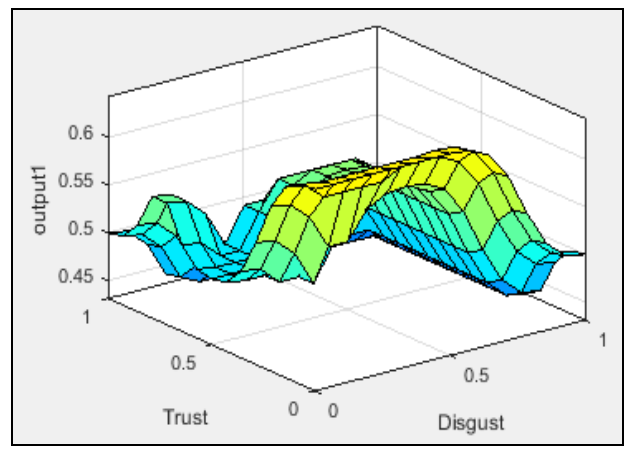

(b)

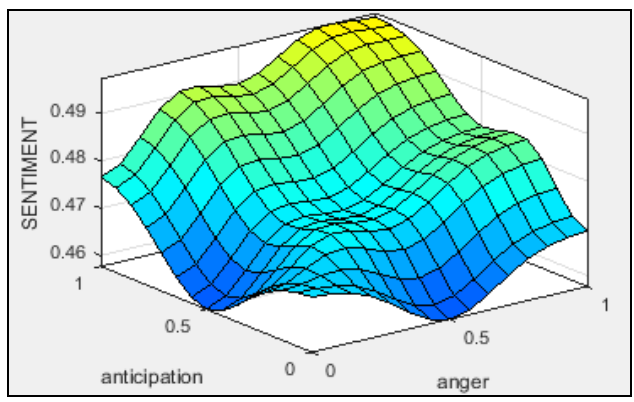

(c)

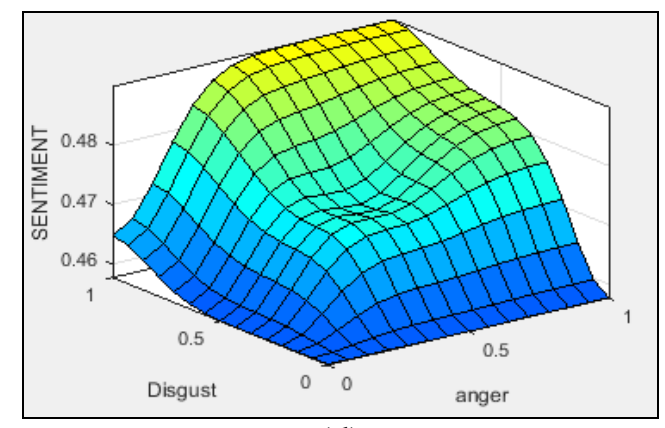

(d)

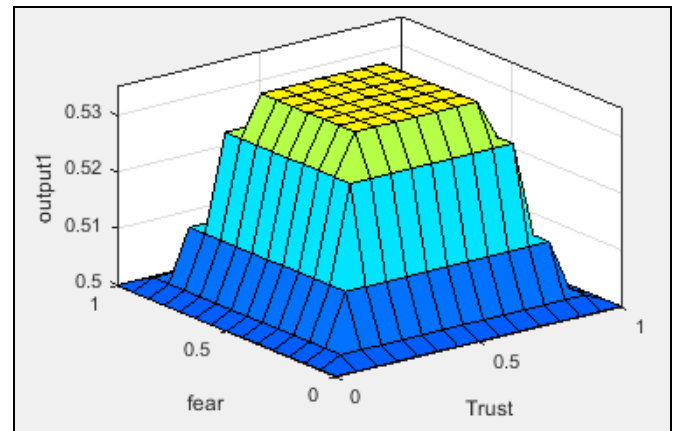

(e)

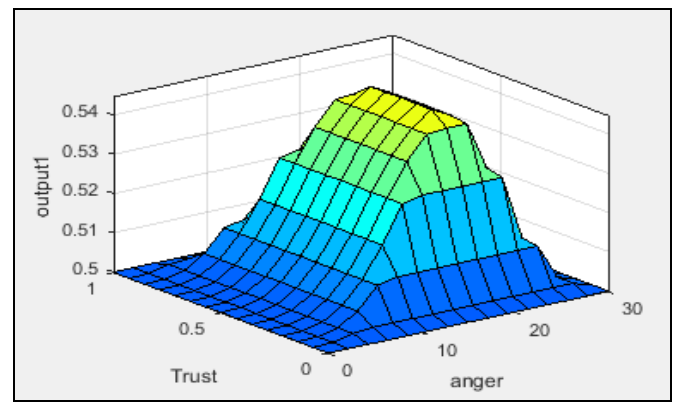

(f)

Figure 4. Plots of Combination of Emotions.

\section{Performance Evaluation}

In Table 5 we have summarized the values obtained using a generic function to produce result summaries of the results of various model fitting functions. 
Table 5. Summary of Machine Learning Algorithm Performance

\begin{tabular}{|l|l|l|l|l|l|}
\hline & SVM & TREE & MAXENTROPY & FOREST & BAGGING \\
\hline PRECISION & 0 & 0.04 & 1 & 1 & 1 \\
\hline RECALL & 0 & 0.2 & 1 & 1 & 1 \\
\hline FSCORE & 0 & 0.066 & 1 & 1 & 1 \\
\hline
\end{tabular}

\section{Validation of Models}

Here we are performing n-fold cross-validation for specified algorithm, with different possible $n$ values.

Table 6. Cross-Validation with $\mathrm{N}=2$

\begin{tabular}{|l|l|l|l|l|l|l|l|}
\hline $\mathrm{N}=2$ & NNET & TREE & FOREST & BOOSTING & SVM & BAGGING & MAXNET \\
\hline Sample & 0125 & 0.142 & 0.473 & 0.68 & 0.619 & 0.684 & 1 \\
Accuracy & 0.062 & 0.105 & 0.428 & 0.60 & 0.684 & 0.619 & 1 \\
\hline $\begin{array}{l}\text { Mean } \\
\text { Accuracy }\end{array}$ & 0.093 & 0.124 & 0.451 & 0.64 & 0.651 & 0.651 & 1 \\
\hline
\end{tabular}

Table 7. Cross-Validation with $\mathrm{N}=5$

\begin{tabular}{|l|l|l|l|l|l|l|}
\hline $\mathrm{N}=5$ & NNET & BAGGING & SVM & RF & BOOSTING & MAXNET \\
\hline & 0.090 & 0.6 & 0.555 & 0.714 & 1 & 1 \\
Sample & 0.090 & 0.555 & 0.8 & 1 & 1 & 1 \\
Accuracy & 0 & 0.714 & 0.75 & 1 & 1 & 1 \\
& 0 & 0.428 & 0.555 & 0.454 & 0.75 & 1 \\
& 0 & 1 & 1 & 0.777 & 0.77 & 1 \\
\hline Mean & 0.036 & 0.659 & 0.732 & 0.789 & 1 & 1 \\
Accuracy & & & & & & \\
\hline
\end{tabular}

\section{Discussion}

The aim of our research work is to construct rules to classify the sentiments using emotions as features by manually assigning weights based on the impact of the emotion on particular tweet. We identified six possible combinations that other research papers had not focused till date. They are like: Trust with $\{\mathrm{Sad}$, Disgust, Fear, Anger $\}$ and Anger with \{anticipation, Disgust . This combination are considered in our experiment, that effect the overall sentiment analysis.

1. In 7(a) we can interpret that Trust and sad combined together has impact of 0.6 on overall sentiment analysis.

2. In 7(b) we can interpret that Trust and Disgust combined together has impact of 0.6 on overall sentiment analysis.

3. In 7(c) we can interpret that anticipate and anger combined together has impact of 0.49 on overall sentiment analysis.

4. In 7(d) we can interpret that Fear and Trust combined together has impact of 0.53 on overall sentiment analysis.

5. In 7(e) we can interpret that Disgust and anger combined together has impact of 0.48 on overall sentiment analysis.

6. In 7(f) we can interpret that Trust and anger combined together has impact of 0.54 on overall sentiment analysis. 


\section{Conclusion and Future work}

In our research we conducted experiments on the proposed algorithm in scoring an emotion present in tweet and demonstration is carried out using FUZZY application available in MATLAB. And implemented the most commonly used types of FRBSs, namely, Mamdani and sugeno, for classification task. We measure the performance of Machine Learning algorithms using parameters like Precision, Recall, F-score and concluded that MAXENTROPY, BAGGING, RANDOM FOREST outperforms the other Machine Learning algorithms. In addition to that we use Cross Validation method with two and five folds to measure the mean accuracy of the algorithms used in classification and concluded that MAXNET give the mean accuracy value of one in both two and five folds. In Future we would like to use Artificial Neural Fuzzy Inference System application available in MATLAB to train the model.

\section{Acknowledgment}

We would like to thank our VIT University to providing us the all the research facilities requirement for publishing SCI journals. At the same time we would like to thank all the reviewers, who helps us to improve the quality of our paper.

\section{References}

[1] Rob Abbott, Marilyn Walker, Pranav Anand, Jean E Fox Tree, Robeson Bowmani, and Joseph King, "How can you say such things?!?: Recognizing disagreement in informal political argument", Proceedings of the Workshop on Languages in Social Media, Association for Computational Linguistics, (2011).

[2] Apoorv Agarwal, Boyi Xie, Ilia Vovsha, Owen Rambow, and Rebecca Passonneau, "Sentiment analysis of twitter data", Proceedings of the workshop on languages in social media, Association for Computational Linguistics, (2011).

[3] Fotis Aisopos, George Papadakis, and Theodora Varvarigou, "Sentiment analysis of social media content using n-gram graphs", Proceedings of the 3rd ACM SIGMM international workshop on Social media, ACM, (2011).

[4] Dimah H. Alahmadi and Xiao-Jun Zeng, "Ists: Implicit social trust and sentiment based approach to recommender systems", Expert Systems with Applications, vol. 42, no.22, (2015), pp. 8840 - 8849.

[5] Ramnath Balasubramanyan, William W Cohen, Doug Pierce, and David P Redlawsk, "What pushes their buttons?: predicting comment polarity from the content of political blog posts", Proceedings of the workshop on languages in social media, Association for Computational Linguistics, (2011).

[6] Johan Bollen, Huina Mao, and Alberto, "Modeling public mood and emotion: Twitter sentiment and socio-economic phenomena", Proceedings of the ICWSM 2011).

[7] Johan Bollen, Huina Mao, and Xiaojun Zeng, "Twitter mood predicts the stock market", Journal of Computational Science, vol. 2, no. 1, (2011), pp. 1-8.

[8] Felipe Bravo-Marquez, Eibe Frank, and Bernhard Pfahringer., "Building a twitter opinion lexicon from automatically-annotated tweets", Knowledge-Based Systems, vol. 108, no.1, (2016), pp. 65 - 78.

[9] Erik Cambria, Bjorn Schuller, Yunqing Xia, and Catherine Havasi, "New avenues in opinion mining and sentiment analysis", IEEE Intelligent Systems, vol. 28, no. 2, (2013), pp. 15-21.

[10] Xi Chen, Ruibin Geng, and Shun Cai, "Predicting microblog users' lifetime activities - a user-based analysis", Electronic Commerce Re-search and Applications, vol. 14,no. 3,(2015), pp. 150 - 168.

[11] Stephan Gouws, Donald Metzler, Congxing Cai, and Eduard Hovy, "Contextual bearing on linguistic variation in social media", Proceedings of the Workshop on Languages in Social Media, Association for Computational Linguistics, (2011).

[12] Michael Hagenau, Michael Liebmann, and Dirk Neumann, "Automated news reading: Stock price prediction based on financial news using context-capturing features", Decision Support Systems, vol. 55, no. 3, (2013), pp. $685-697$.

[13] Susan C Herring. Automating analysis of social media communication: insights from cmda. Proceedings of the Workshop on Languages in Social Media, Association for Computational Linguistics, (2011).

[14] Jian Jin, Ping Ji, and C.K. Kwong, "What makes consumers unsatisfied with your products: Review analysis at a fine-grained level", Engineering Applications of Artificial Intelligence, vol. 47, no. 1, (2016), pp. $38-48$.

[15] Aamera ZH Khan, Mohammad Atique, and VM Thakare, "Combining lexicon-based and learning-based methods for twitter sentiment analysis", International Journal of Electronics, Communication and Soft Computing Science \& Engineering, (2015). 
[16] Farhan Hassan Khan, Saba Bashir, and Usman Qamar, "Tom: Twitter opinion mining framework using hybrid classification scheme", Decision Support Systems, vol. 57, (2014), pp. 245-257.

[17] Efthymios Kouloumpis, Theresa Wilson, and Johanna D Moore, "Twitter sentiment analysis: The good the bad and the omg!", Proceedings of the International conference, Icwsm, (2011).

[18] B. Shravan Kumar and Vadlamani Ravi, "A survey of the applications of text mining in financial domain", Knowledge-Based Systems, vol. 114, (2016), pp. 128 - 147.

[19] Artur Lugmayr and Gerhard Gossen, "Evaluation of methods and techniques for language based sentiment analysis for dax 30 stock exchange a first concept of a "lugo" sentiment indicator", International SERIES on Information Systems and Management, vol. 1, (2013), pp. 69-76.

[20] Alejandro Maté, Jesús Peral, Antonio Ferrández, David Gil, and Juan Trujillo, "A hybrid integrated architecture for energy consumption prediction", Future Generation Computer Systems, vol. 63, (2016), pp. $131-147$.

[21] Walaa Medhat, Ahmed Hassan, and Hoda Korashy, "Sentiment analysis algorithms and applications: A survey", Ain Shams Engineering Journal, vol. 5, no. 4, (2014), pp. 1093 - 1113.

[22] Saif M. Mohammad. "9 - sentiment analysis: Detecting valence, emotions, and other affectual states from text", In Herbert L. Meiselman, editor, Emotion Measurement. Woodhead Publishing, (2016), pp. $201-237$.

[23] Mohammad SM, Turney PD, "Crowdsourcing a word-emotion association lexicon", Computational Intelligence, vol. 29, no. 3, (2013), pp. 436-65.

\section{Authors}

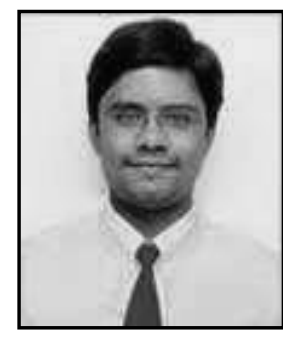

Syed Muzamil Basha, he had his Bachelor of Science in Information Technology at SITAMS, MTech in Information Technology (Networking) at VIT University and currently doing his research at VIT University. His research area are Wireless Sensor Networks, Text Mining and Big Data Predictive Analytics.

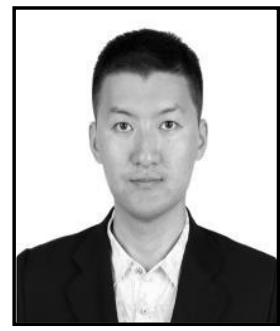

Yang Zhenning, he is pursuing M.ScComputer Science at School of Computing Science and Engineering, VIT University, Vellore. His area of interests are Algorithm design an Pattern Recognition, operating Systems and cloud computing.

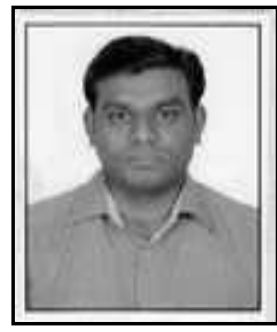

Dharmendra Singh Rajput working as Associate Professor in the Department of Software and Systems Engineering, School of Information Technology and Engineering, VIT University. His research area are Data Mining and Big Data Predictive Analytics.

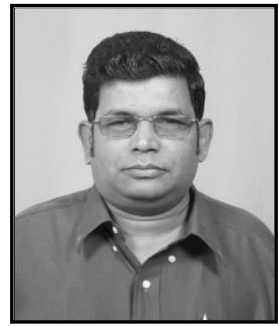

N. Ch. S. N. Iyengar (b 1961), he currently Senior Professor at the School of Computer Science and Engineering at VIT University, Vellore-632014, Tamil Nadu, India. His research interests include Agent-Based Distributed Computing, Intelligent Computing, Network Security, Secured Cloud Computing and Fluid Mechanics. $\mathrm{He}$ had $30+$ years of experience in teaching and research, guided 


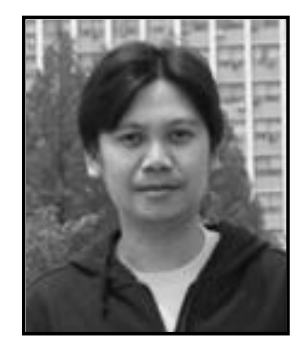

many scholars, has authored several textbooks and had nearly $200+$ research publications in reputed peer reviewed international journals. $\mathrm{He}$ served as $\mathrm{PCM} / \mathrm{reviewer} /$ keynotespeaker/Invited speaker for many conferences. He serves as editorial board member for many international journals, reviews papers for many conferences with an interest of serving to the education community

Ronnie D. Caytiles, he had his Bachelor of Science in Computer Engineering- Western Institute of Technology, Iloilo City, Philippines, and Master of Science in Computer Science- Central Philippine University, Iloilo City, Philippines. He finished his Ph.D. in Multimedia Engineering, Hannam University, Daejeon, Korea. Currently, he serves as an Assistant Professor at Multimedia Engineering department, Hannam University, Daejeon, Korea. His research interests include Mobile Computing, Multimedia Communication, Information Technology Security, Ubiquitous Computing, Control and Automation. 